\title{
Pênfigo vulgar na cavidade bucal: relato de caso clínico
}

\author{
Pemphigus vulgaris in oral cavity: clinical case report
}

Danielly de Fátima Castro Leite*

Mauricio Pereira Macedo**

Camila Maria de Sousa Simas ${ }^{* * *}$

Luana Carneiro Diniz Souza****

Fernanda Ferreira Lopes ${ }^{* * * *}$

\section{Resumo}

O pênfigo vulgar é uma doença autoimune que pode ter um prognóstico grave. As manifestações bucais são frequentemente os primeiros sinais da doença. Objetivo: apresentar um caso clínico de pênfigo vulgar em uma paciente de 29 anos de idade, atendida no Serviço de Odontologia do Hospital Universitário da Universidade Federal do Maranhão, São Luis, Maranhão. Relato de caso: paciente com o diagnóstico de pênfigo vulgar, queixando-se de lesões dolorosas na cavidade bucal e na pele, com evolução de três semanas. Na admissão hospitalar, além das lesões de pênfigo vulgar, a paciente apresentava depressão, diabetes descompensada, osteoporose e úlcera gástrica. A paciente recebeu o tratamento multiprofissional e foi medicada para o controle do pênfigo e das outras patologias, e com a melhora significativa do quadro clínico, recebeu alta. Considerações finais: o presente relato de caso clínico contribuiu para ampliar o conhecimento do cirurgião-dentista na abordagem odontológica e conduta multiprofissional em relação ao pênfigo vulgar.

Palavras-chave: Pênfigo. Saúde bucal. Diagnóstico.

\section{Introdução}

As doenças dermatológicas autoimunes são condições patológicas decorrentes da ativação do sistema imunológico contra elementos teciduais, particularmente da pele ou de superfícies das mucosas ${ }^{1}$. Dentre as dermatoses mais estudadas, podemos enfatizar o pênfigo, que é uma patologia imunologicamente mediada e caracterizada pela formação de bolhas intraepiteliais na pele e em mucosas ${ }^{2}$.

O pênfigo vulgar é o tipo de pênfigo mais comum, mesmo assim ele não é observado com muita frequência. A forma vulgar pode ter um prognóstico grave, devido à possibilidade de seguir um curso clínico preocupante quando não diagnosticada e tratada inicialmente ${ }^{3}$. A patologia acomete com maior frequência indivíduos na idade adulta, entre a quinta e sexta décadas de vida, é particularmente rara em crianças e idosos, não foi observada nenhuma predileção por gênero, embora alguns estudos mostrem prevalência no gênero feminino ${ }^{4}$. 
A etiologia do pênfigo vulgar não é totalmente definida, mas pode ter a possibilidade da predisposição genética com forte associação dos alelos HLA (DRBI*0402 e DQBI*0503), além dos fatores endógenos (alterações imunológicas), fatores exógenos (drogas, infecções, neoplasias e agentes físicos) e grupos raciais (judeus e descendentes da região mediterrânea $)^{5}$. Suas manifestações acometem a cavidade bucal e frequentemente são o primeiro sinal da doença. As alterações são caracterizadas pela persistência e duração maior do que as lesões cutâneas ${ }^{6}$.

O presente trabalho tem por objetivo apresentar um caso clínico de pênfigo vulgar bem como a conduta clínica multiprofissional adequada, visando contribuir com o conhecimento sobre o papel do cirurgião-dentista em relação a essa patologia.

\section{Relato de caso}

Paciente do gênero feminino, feoderma, 29 anos de idade, com o diagnóstico de pênfigo vulgar desde 2011, foi internada no Hospital Universitário Presidente Dutra, da Universidade Federal do Maranhão (UFMA), queixando-se de lesões dolorosas no corpo e na cavidade bucal. Em relação ao aspecto ético, este estudo obteve aprovação por meio do Parecer no 981.721/2015, do Comitê de Ética em Pesquisa da UFMA. A paciente relatou que as lesões tinham evolução de três semanas, afetando inicialmente a mucosa bucal, depois os membros superiores, inferiores e tronco, associadas à sensibilidade dolorosa tipo queimação e de grande intensidade.

A paciente apresentava depressão, diabetes descompensada, osteoporose, úlcera gástrica e quadro não controlado de pênfigo vulgar. No controle das patologias, foram prescritos os medicamentos no período da internação: prednisona $80 \mathrm{mg} /$ dia (pênfigo vulgar), azatioprina $100 \mathrm{mg} /$ dia (pênfigo vulgar), tramadol IV de $8 / 8 \mathrm{~h}$ (analgesia), alendronato de sódio $70 \mathrm{mg} /$ dia (osteoporose), diazepan $5 \mathrm{mg} / \mathrm{dia}$ (depressão), amitriptilina $25 \mathrm{mg} /$ dia (depressão), ranitidina IV de $8 / 8 \mathrm{~h}$ (úlcera gástrica) e o esquema insulinoterapia (Insulina NPH) e metformina 850 $\mathrm{mg} /$ dia (diabetes).

A equipe médica solicitou um parecer para o setor de odontologia do hospital para avaliação da paciente. Durante anamnese, a paciente queixou-se de dificuldade na mastigação devido às lesões. Ela relatou que primeiramente surgiram bolhas na cavidade bucal, que se romperam rapidamente, dando lugar a ulcerações dolorosas.

O exame físico extrabucal revelou a presença de lesões eritematosas descamativas na região geniana, mentoniana e labial com ausência de linfoadenopatia cervical (Figura 1). No exame intrabucal, observou-se a presença de lesões de halo eritematoso na região do palato e ventre da língua, e uma pseudomembrana na região do dorso lingual (Figu- ras 2,3 e 4). A saúde bucal da paciente era insatisfatória, com a presença de cálculo dental, raízes residuais dos elementos 17,16 e 28 e lesões cariosas nos elementos 18, 32, 31, 43 e 44 . O quadro geral instável da paciente exigiu diferentes abordagens de tratamento multiprofissional.

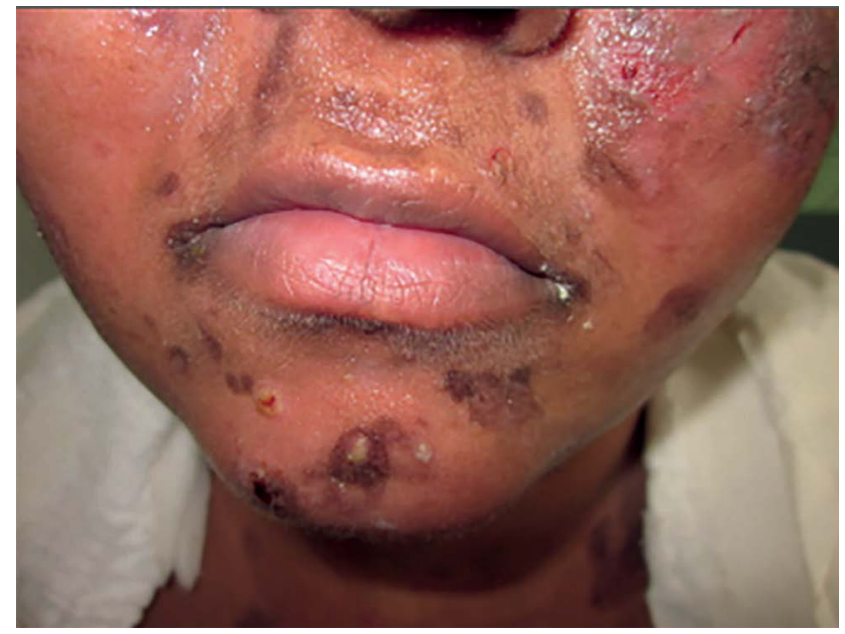

Figura 1 - Exame físico extrabucal

Fonte: todas as figuras são de elaboração dos autores.

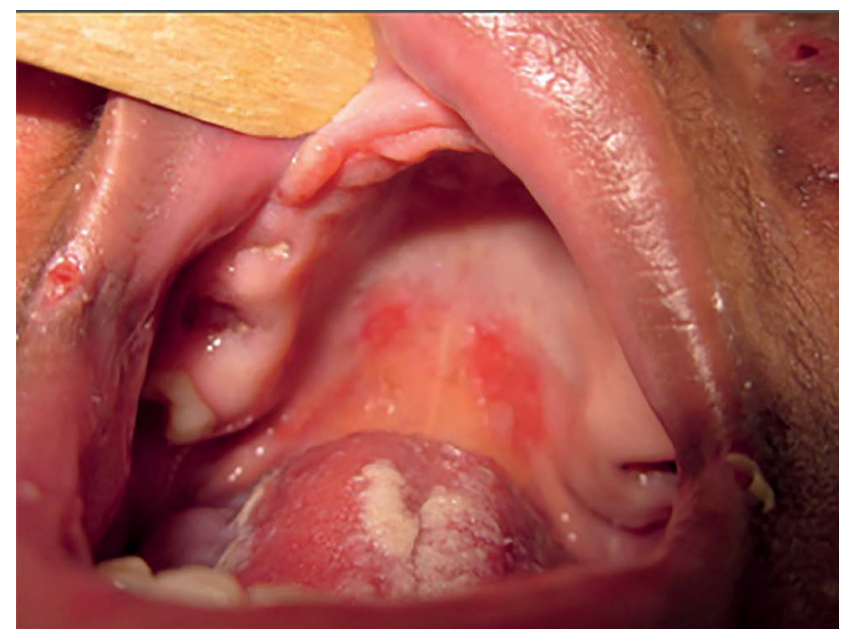

Figura 2 - Exame físico intrabucal - lesão na região do palato e no dorso da língua

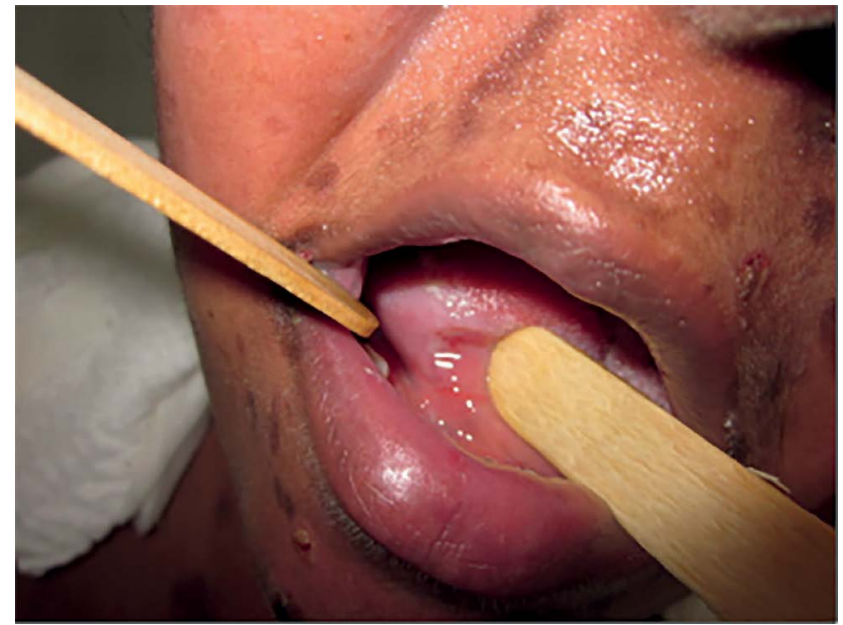

Figura 3 - Exame físico intrabucal - lesão na região do ventre da língua 


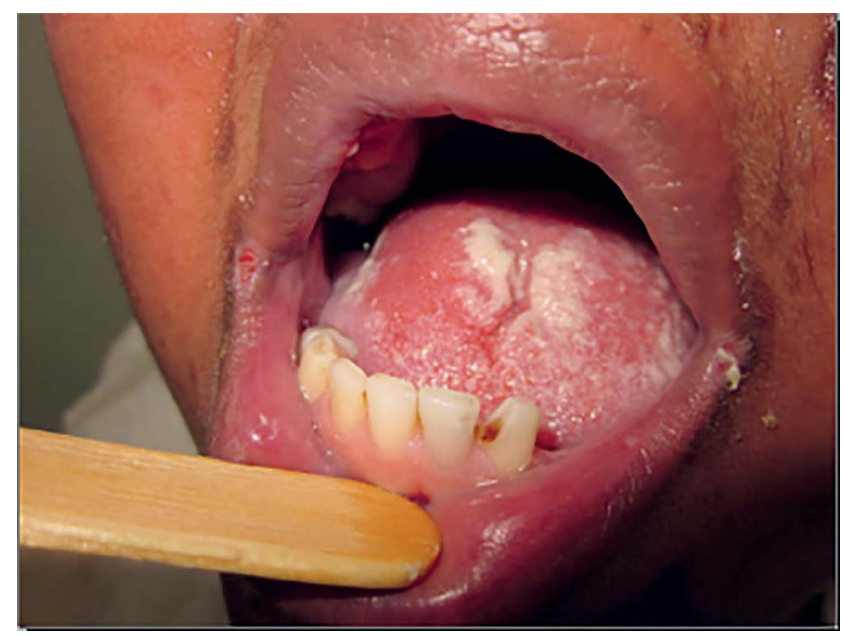

Figura 4 - Exame físico intrabucal - lesão na região do dorso da língua

Para o controle do pênfigo, a prescrição médica consistiu em elevadas doses de corticosteroide (prednisona, de $80 \mathrm{mg}$ a $120 \mathrm{mg}$ diários) e mais o agente imunossupressor (azatioprina $100 \mathrm{mg} / \mathrm{dia}$ ). As outras alterações, como depressão, diabetes, osteoporose e úlcera gástrica, eram efeitos colaterais potencialmente associados ao uso de longo prazo do corticosteroide sistêmico. Essas alterações foram controladas pelos medicamentos prescritos e concomitantemente pela atuação do psicólogo e do terapeuta ocupacional que acompanharam o caso.

A equipe de odontologia prescreveu acetonida de triancinolona $1 \mathrm{mg}$ em base emoliente para uso tópico nas lesões da mucosa bucal por quinze dias, além da execução do tratamento periodontal, restaurações, exodontia das raízes residuais e orientação da higiene bucal (clorexidina 0,12\% sem álcool e escova dental extramacia).

Os cuidados de enfermagem implementados foram de orientações em relação à doença e seus cuidados, ao apoio emocional e à realização diária do curativo com sulfadiazina de prata após banho de aspersão com ringer simples e clorexidina nas lesões de pele.

Durante a internação, na análise da urocultura da paciente, foi detectada a bactéria Escherichia coli, e no material colhido da lesão de pele, houve o crescimento das bactérias Acinetobacter baumannii e Pseudomonas aeruginosa. Na cultura da secreção nasal, foram constatadas evidências de crescimento das bactérias Staphylococcus sp. coagulase negativa e Staphylococcus aureus. Os resultados das hemoculturas e da cultura das lesões bucais foram normais. Com base nos resultados das culturas, foram administrados antibióticos no período de internação seguindo os critérios de sensibilidade dos micro-organismos e as normas da Comissão de Controle de Infecção Hospitalar.

Após a alta hospitalar, a paciente voltou para sua cidade de origem. Durante os meses que seguiram, a paciente compareceu ao hospital para o tratamento de pulsoterapia (metilprednisolona) para o controle do pênfigo vulgar. Retornando ao setor de odontologia, a paciente relatou ausência de qualquer sintomatologia na pele e na cavidade bucal, e no exame físico todas as estruturas apresentavam aspectos de normalidade. $\mathrm{O}$ tratamento periodontal foi realizado e a paciente recebeu orientação para a confecção da prótese dentária parcial em sua cidade de origem.

\section{Discussão}

O pênfigo vulgar é uma doença autoimune rara, e sua incidência varia de 0,2 a 3,2 casos em cada 100.000 indivíduos/ano, principalmente em adultos entre a quinta e sexta décadas de vida. Apesar de sua baixa ocorrência, a forma vulgar pode ter um prognóstico grave, entretanto, os índices elevados de mortalidade associados ao pênfigo vulgar foram radicalmente reduzidos desde a introdução de corticosteroides $^{3}$. Em aproximadamente $50 \%$ dos casos, os sinais da doença tem início na mucosa bucal, antes das lesões da pele e outras mucosas (nariz, faringe, laringe e esôfago $)^{7}$, como ocorreu no caso aqui relatado.

$\mathrm{Na}$ paciente acompanhada neste estudo, a doença teve um curso rápido e agressivo, posteriormente às lesões na cavidade bucal, apareceram as lesões cutâneas. Achado que corrobora com os resultados de pesquisas em pacientes com o pênfigo vulgar que apresentaram os primeiros sinais da doença na mucosa bucal ${ }^{8,9}$. Além disso, a paciente apresentou lesões na região do palato e ventre da língua e presença de pseudomembrana na região do dorso lingual. Estudos afirmam ser a mucosa bucal, o palato e a gengiva os locais mais acometidos ${ }^{10}$.

Alguns autores afirmam que a forma vulgar é o tipo de pênfigo mais predominante ${ }^{11}$. Em um estudo realizado para avaliar os casos de pênfigo, de cinquenta pacientes, 31 foram diagnosticados com pênfigo vulgar, dezesseis com pênfigo foliáceo, dois com pênfigo paraneoplásico e um com pênfigo $\operatorname{IgA}^{12}$. Outra pesquisa corrobora com esse achado: foram 38 casos de pênfigo vulgar com o comprometimento na cavidade bucal e somente doze casos apresentaram lesões cutâneas e/ou da mucosa ocular ${ }^{13}$.

O diagnóstico do pênfigo vulgar é baseado nos achados clínicos e histopatológicos e na técnica de anticorpos fluorescentes do tecido da biópsia. Nessa técnica ocorre a visualização da separação intraepitelial, acantólise da camada espinhosa e presença das células de Tzanck, manifestando-se clinicamente na forma de bolhas na mucosa bucal e na pele, devido à reação dos autoanticorpos contra as desmogleínas 1 e $3^{14}$.

No caso clínico relatado, a paciente com o diagnóstico de pênfigo vulgar já há um ano relatou o aparecimento de bolhas que precederam as ulcerações presentes na cavidade bucal e na pele. O tratamento utilizado nesse caso foi o corticoide sistêmico 
prednisona, medicamento que a paciente já utilizava, mantendo-se assim o seu plano terapêutico.

A prednisona é o medicamento de escolha para a maioria dos $\operatorname{casos}^{15}$. Em um estudo que utilizou semelhante tratamento, a prednisona foi opção em dezoito pacientes (78\%) e o deflazacort foi usado em apenas cinco $(22 \%)^{16}$. A morbidade e a mortalidade de pacientes com pênfigo vulgar ocorrem devido aos efeitos da terapia corticoide prolongada. Em contrapartida, o óbito era de $30 \%$ devido ao desequilíbrio eletrolítico, e a sepse diminuiu para $6 \%$ com a introdução da terapia corticoide ${ }^{17}$.

$\mathrm{Na}$ literatura, recomenda-se o uso do corticoide sistêmico em doses elevadas com diminuição progressiva até dose de manutenção com controle clínico e laboratorial visando à diminuição dos efeitos secundários ${ }^{18}$. A dose inicial da prednisona $(80 \mathrm{mg}$ a $120 \mathrm{mg}$ diários) para o caso clínico em questão foi inicialmente elevada, objetivando o controle da doença. Além disso, foi prescrito o imunossupressor azatioprina (100 mg/dia) para redução dos efeitos colaterais da terapia com corticoide sistêmico. A remissão do pênfigo é lenta e variável para cada paciente. A taxa de mortalidade diminui se houver precocidade do diagnóstico. Assim, quando o tratamento é iniciado, menores serão as doses de corticoides sistêmicos reduzindo as reações adversas ${ }^{19}$.

Aplicação do corticoide tópico (cremes, pastas) pode permitir o uso de doses sistêmicas inferiores, e nos casos resistentes à terapêutica oral, deverá ser considerada a pulsoterapia com metilprednisolona ou ciclofosfamida intravenosa em altas $\operatorname{doses}^{20}$. A paciente apresentava lesões ulceradas na cavidade bucal e esteve medicada com o corticoide tópico acetonida de triancinolona concomitantemente com a terapia sistêmica. Após a alta hospitalar e com a remissão completa das lesões bucais e da pele, utilizou-se corticoterapia sistêmica oral e a terapia adjuvante (pulsoterapia).

Em um estudo, 31 pacientes com o diagnóstico de pênfigo vulgar receberam doses de prednisona para o controle da doença, e 27 deles receberam terapia adjuvante. A dapsona foi o medicamento de escolha para vinte pacientes, azatioprina para cinco pacientes, e dois pacientes receberam ciclofosfami$\mathrm{da}^{21}$. Outra terapêutica adjuvante é a imunoglobulina intravenosa e a utilização de diferentes imunossupressores para diminuir a dose de corticosteroide, outras alternativas são o rituximab, anticorpo monoclonal antiCD20 dirigido aos linfócitos B e à plasmaferese ${ }^{22}$.

O uso prolongado do corticoide pode trazer reações adversas, como obesidade, diabetes, osteoporose e outros distúrbios, por isso, a utilização de imunossupressores, como azatioprina, ciclofosfamida e micofenolato de metila, visa diminuir os efeitos adversos ${ }^{23}$. Na admissão hospitalar, a paciente tinha um quadro descontrolado de pênfigo vulgar, além de apresentar os efeitos adversos potencialmente associados à utilização em longo prazo de corticoi- des sistêmicos, efeitos que se manifestaram como depressão, diabetes, osteoporose e úlcera gástrica.

O acompanhamento do caso evidenciou a importância da abordagem multiprofissional devido ao descontrole da patologia e a outros problemas que a paciente apresentou no período da internação. $\mathrm{O}$ atendimento odontológico, associado aos cuidados da enfermagem e à administração de corticoides sistêmicos, e o controle do diabetes, da osteoporose, da depressão e das infecções colaboraram para a melhora do quadro clínico geral e bucal da paciente, o que permitiu a alta hospitalar e a proservação da paciente.

\section{Considerações finais}

O pênfigo vulgar é uma patologia autoimune rara que ocorre principalmente na idade adulta. $\mathrm{O}$ diagnóstico é baseado em características clínicas e histopatológicas. Clinicamente, o pênfigo manifesta-se em forma de bolhas, característica observada histopatologicamente com a separação intraepitelial. A forma de tratamento indicada é a corticoterapia sistêmica com controle clínico e laboratorial para evitar a exacerbação da doença. O presente relato de caso clínico contribuiu para ampliar o conhecimento do cirurgião-dentista em relação ao pênfigo vulgar, enfatizando a abordagem odontológica e a conduta multiprofissional.

\section{Abstract}

Pemphigus vulgaris is an autoimmune disease that may present a severe prognosis. Oral manifestations are often the first signs of the disease. Objective: To present a clinical case of pemphigus vulgaris in a 29-year-old patient treated at the Dental Service of the University Hospital of the Federal University of São Luis, Maranhão, Brazil. Case report: Patient diagnosed with pemphigus vulgaris claiming painful lesions in the oral cavity and skin, with a three-week evolution. On hospital admission, besides pemphigus vulgaris lesions, the patient presented depression, decompensated diabetes, osteoporosis, and gastric ulcer. The patient received multidisciplinary treatment and medication to control pemphigus and other conditions; upon significant improvement of symptoms, the patient was discharged. Final considerations: The present clinical case contributed to broaden the understanding of the dentist on the dental approach and the multidisciplinary conduct regarding pemphigus vulgaris.

Keywords: Pemphigus. Oral health. Diagnosis. 


\section{Referências}

1. Schmidt E, Zillikens D. Diagnosis and treatment of patients with autoimmune bullous disorders in Germany. Dermatol Clin 2011; 29(4):663-71.

2. Scully C, Mignogna M. Oral mucosal disease: pemphigus. $\mathrm{Br}$ J Oral Maxillofac Surg 2008; 46(4):272-7.

3. Ferreira FA, Filippini PA, Beltrame M, Guirra FR, Barreto MP. Manifestações bucais dos pênfigos vulgar e bolhoso. Odontol Clín-Cient 2009; 8(4):293-8.

4. Bascones-Martínez A, García-García V, Meurman JH, Requena-Caballero L. Immune-mediated diseases: what can be found in the oral cavity? Int J Dermatol 2015; 54(3):258-70.

5. Stoopler ET, Sollecito TP. Oral mucosal diseases: evaluation and management. Med Clin North Am 2014; 98(6):1323-52.

6. Ohta M, Osawa S, Endo H, Kuyama K, Yamamoto H, Ito T. Pemphigus vulgaris confined to the gingiva: a case report. Int J Dent 2011; 2011:1-4.

7. Tan SR, Mc Dermott MR, Castillo CJ, Sauder DN. Pemphigus vulgaris induced by electrical injury. Cutis 2006; 77(3):161-5.

8. Chmurova N, Svecova D. Pemphigus vulgaris: a 11-year review. Bratisl Lek Listy 2009; 110(8):500-3.

9. Karagöz G, Bektaş-Kayhan K, Ünür M. Evaluation of Pemphigus cases involving oral mucosa. OHMD 2014; 13(3):605-9.

10. Ruocco V, Ruocco E, Lo Schiavo A, Brunetti G, Guerrera LP, Wolf R. Pemphigus: etiology, pathogenesis, and inducing or triggering. Clin Dermatol 2013; 31(4):374-8.

11. Heelan K, Mahar AL, Walsh S, Shear NH. Pemphigus and associated comorbidities: a cross-sectional study. Clin Exp Dermatol 2015; 40(6):593-9.

12. Goon AT, Tan SH. Comparative study pemphigus vulgaris and pemphigus foliaceus in Singapore. Australas J Dermatol $2001 ; 42(3): 172-5$.

13. Moleri AB, Jordão MB, Ribeiro DMC, Moreira LC. Lesões orais do pênfigo vulgar: a importância do diagnóstico precoce. Acta Scientia e Medica 2008; 2(1):72-9.

14. Cunha PR, Barraviera SRCS. Dermatoses bolhosas autoimunes. An Bras Dermatol 2009; 84(2):111-24.

15. Endo H, Rees TD, Hallmon WW, Kuyama K, Nakadai $\mathrm{M}$, Kato $\mathrm{T}$ et al. Disease progression from mucosal to $\mathrm{mu}$ cocutaneous involvement in a patient with desquamative gingivitis associated with pemphigus vulgaris. J Periodontol 2008; 79(2):369-75.

16. Miziara ID, Ximenes Filho JA, Ribeiro FC, Brandão AL. Acometimento oral no pênfigo vulgar. Rev Bras Otorrinolaringol 2003; 3(69):327-31.

17. Qasmi S, Aoussar A, Senouci K, Khabbal Y, Abouqual R, Hassam $\mathrm{B}$ et al. Impact of oral lesions and diagnosis, treatment and prognosis of pemphigus. JEADV 2009; 23(4):46970 .

18. Toth GG, Jonkman MF. Therapy of Pemphigus. Clin Dermatol 2001; 6(19):761-7.

19. Dagistan S, Goregen M, Miloglu O, Cakur B. Oral pemphigus vulgaris: a case report with review of the literature. $\mathrm{J}$ Oral Sci 2008; 50(3):359-62.

20. Femiano F, Gombos F, Scully C. Pemphigus vulgaris with oral involvement: evaluation of two different systemic corticosteroid therapeutic protocols. JEADV 2002; 16(4):353-6.

21. Ahmed AR, Dahl MV. Consensus statement on the use of intravenous immunoglobulin therapy in the treatment of autoimmune mucocutaneous blistering diseases. Arch Dermatol $2003 ; 139(8): 1051-9$
22. Cianchini G, Corona R, Frezzolini A, Ruffelli M, Didona B, Puddu P. Treatment of severe Pemphigus with rituximab: report of 12 cases and a review of the literature. Arch Dermatol 2007; 143(8):1033-8.

23. Kapoor S, Sikka P, Kaur GP. Pemphigus vulgaris of oral cavity: a case report with its treatment strategies. Int J Nutr Pharmacol Neurol Dis 2013; 2(3):146-9.

\section{Endereço para correspondência:}

Danielly de Fátima Castro Leite

Avenida da História, Bloco A1, apto. 301

Cohafuma

65074-795 São Luis Maranhão, MA Brasil

Telefone: (98) 98812-0594

E-mail: danielly.leite505@gmail.com

Recebido: 19/08/15. Aceito: 21/09/15. 